\title{
Bijective proofs involving chromatic overpartitions
}

\author{
Mateus Alegri \\ Department of Mathematics (DMAI), University of Sergipe \\ 49500-000, Itabaiana-SE, Brazil \\ e-mail: allegri.mateus@gmail.com
}

Received: 23 May 2018

Accepted: 18 December 2018

\begin{abstract}
In this paper, our aim is to provide two bijective proofs for identities involving what we call chromatic overpartitions, which is a generalization of the well-known overpartitions class. For this purpose we will give the mathematical definitions of chromatic overpartitions, providing their respective generating functions.
\end{abstract}

Keywords: Integer partitions, Overpartitions, Chromatic partitions, Partition identities.

2010 Mathematics Subject Classification: Primary 05A17, Secondary 11P82, 11P84.

\section{Introduction}

In this paper, we use the following notation.

$$
(a, q)_{n}= \begin{cases}(1-a)(1-a q)\left(1-a q^{2}\right) \cdots\left(1-a q^{n-1}\right), & \text { if } n>0 \\ 1, & \text { if } n=0\end{cases}
$$

Taking the limit $n \rightarrow \infty$, we have:

$$
(a, q)_{\infty}=\lim _{n \rightarrow \infty}(a, q)_{n}
$$

In [1], we use combinatorial arguments to prove identities like as identity (1), shown below.

$$
\sum_{n=0}^{\infty} \frac{n q^{n}}{(1-q)(1-2 q) \cdots\left(1-n q^{n}\right)}=\prod_{n=1}^{\infty} \frac{1}{\left(1-n q^{n}\right)} .
$$

Indeed, we proved (1) by means of a new class of partitions, namely, the chromatic partitions. The new class of chromatic partitions comes from the necessity for generalization of ordinary 
partitions, due to the fact that the terms $\left(1-k q^{j}\right)^{-1}$, for $|q|<1$ and a positive integer $k$, appear in the equations like (1). In order to find a meaning for these terms, we managed to enumerate the coefficients of $q^{n}$ appearing in the product, using the trivial concept of permutations with repetitions. For example, considering the product

$\frac{1}{1-2 q^{2}} \cdot \frac{1}{1-5 q^{5}}=\left(1+2 q^{2}+2^{2} q^{4}+2^{3} q^{6}+2^{4} q^{8}+2^{5} q^{10}+\ldots\right)\left(1+5 q^{5}+5^{2} q^{10}+5^{3} q^{15}+\ldots\right)$,

the coefficient of $q^{10}$ is equal to 57 , where this number is obtained from

$$
32 q^{2+2+2+2+2}+25 q^{5+5} .
$$

We can interpret the sum in terms of integer partitions that there are 32 partitions of type $2+2+2+2+2$ and 25 of type $5+5$ in this product. Our immediate goal is to distinguish between partitions of each type using colours. If we count with seven colours: red, yellow, green, blue, indigo and violet, the number 2 receives the colours red and yellow, and the number 5 receives the other five colours, and if the order of presentation of the numbers matter, by the multiplicative principle we have $2^{5}=32$ coloured partitions of $2+2+2+2+2$ and $5^{2}=25$ coloured partitions of $5+5$.

Our goal in this work is to find some bijective proofs for new chromatic overpartitions identities. Bijective proofs involving partition identities are widely used to establish simple results as found in [3], [4], and discussed in [2] and [8]. The importance of this type of proof is due to the fact of making a bijective association between one class of partition to another, using simple invertible operation, without the need to establish a generating function for both.

In this work we use the concept of the overpartition of an integer $n$, defined in [5] an discussed in [6] and [7]. The generating function of the ordinary overpartitions, is given by

$$
\sum_{n=0}^{\infty} \frac{(-1, q)_{n} q^{n}}{(q, q)_{n}}=\frac{(-q, q)_{\infty}}{(q, q)_{\infty}} .
$$

From the above identity we can easily obtain the identity below.

$$
\frac{(-q, q)_{\infty}}{(q, q)_{\infty}}=\frac{1}{(q, q)_{\infty}\left(q, q^{2}\right)_{\infty}} .
$$

The right-hand side of the equation (3) is the generating function for partitions in which the parts appear with an index: odd parts appear with indices 0 or 1 , and the even parts are indexed by 0 . The order of the indexed parts should be disgarded. Thus the number of overpartitions of a positive integer $n, \bar{p}(n)$ is equal to the number of indexed partitions by 0 or 1 (or partitions with attached parts), wherein each odd part receives the index 0 or 1 and the even parts are indexed by 0 . We can obtain a bijective proof to this fact. Considering $A_{n}$ the set of partitions of $n$ indexed by 0 or 1 with the restrictions mentioned above, and $O_{n}$ the set of all overpartitions of $n$, the function $f: A_{n} \rightarrow O_{n}$ defined by the rule below is bijective.

- A part with index 0 is associated with a non-overlined part;

- A part with index 1 is associated with an overlined part. But every two odd equal parts with index 1 , of type $\lambda_{1}+\lambda_{1}$, are associated to $2 \overline{\lambda_{1}}$. 
In this work, we will provide some generalizations inspired by this bijective proof.

The next table shows the association 1-1 for the case $n=4$.

\begin{tabular}{|c|c|c|c|c|c|}
\hline \hline Overpartition & & Indexed partition & Overpartition & & Indexed partition \\
\hline \hline 4 & $\leftrightarrow$ & $4_{0}$ & $2+2$ & $\leftrightarrow$ & $2_{0}+2_{0}$ \\
\hline$\overline{4}$ & $\leftrightarrow$ & $1_{1}+1_{1}+1_{1}+1_{1}$ & $\overline{2}+2$ & $\leftrightarrow$ & $2_{0}+1_{1}+1_{1}$ \\
\hline $3+1$ & $\leftrightarrow$ & $3_{0}+1_{0}$ & $2+1+1$ & $\leftrightarrow$ & $2_{0}+1_{0}+1_{0}$ \\
\hline$\overline{3}+1$ & $\leftrightarrow$ & $3_{1}+1_{0}$ & $\overline{2}+1+1$ & $\leftrightarrow$ & $1_{0}+1_{0}+1_{1}+1_{1}$ \\
\hline $3+\overline{1}$ & $\leftrightarrow$ & $3_{0}+1_{1}$ & $2+\overline{1}+1$ & $\leftrightarrow$ & $2_{0}+1_{1}+1_{0}$ \\
\hline$\overline{3}+\overline{1}$ & $\leftrightarrow$ & $3_{1}+1_{1}$ & $\overline{2}+\overline{1}+1$ & $\leftrightarrow$ & $1_{0}+1_{1}+1_{1}+1_{1}$ \\
\hline $1+1+1+1$ & $\leftrightarrow$ & $1_{0}+1_{0}+1_{0}+1_{0}$ & $\overline{1}+1+1+1$ & $\leftrightarrow$ & $1_{1}+1_{0}+1_{0}+1_{0}$ \\
\hline \hline
\end{tabular}

Table 1. Bijective association between overpartitions and indexed partitions.

\section{Background}

In this section we give a few definitions and state a proposition that is the base for constructing the bijections.

Definition 1. A $(n, k)$-chromatic partition is an integer partition of $n$ in which every part receives an index, ranging from 0 to $k-1$, referring to colours. The order of appearance of coloured parts should be considered, i.e., equal parts associated to different colours are considered distinct.

Definition 2. For positive integers $n$ and $k$, an $(n, k)$-super-chromatic partition is a chromatic partition of $n$ where each part $j$ can appear with up to $k^{j}$ different colours.

\section{Example 1.}

- $5_{4}+3_{2}+3_{7}+2_{0}+1_{8}$ is a (21,9)-chromatic partition distinct from $5_{4}+3_{7}+3_{2}+2_{0}+1_{8}$.

- $5_{40}+5_{32}+4_{15}+3_{7}+2_{5}+1_{0}$ is a (20,2)-super-chromatic partition.

- For $n=k=4,4_{a}, 3_{a}+1_{b}, 2_{a}+2_{b}, 2_{a}+1_{b}+1_{c}, 1_{a}+1_{b}+1_{c}+1_{d}$ are indexed partitions, and taking colours $a, b, c$ and $d$, where $0 \leq a, b, c, d \leq 3$, there are $356(4,4)$-chromatic partitions.

For $|k q|<1$, the infinite products $(k q, q)_{\infty}^{-1}$, and $(k q, k q)_{\infty}^{-1}$ are the generating functions for the number of chromatic partitions and super-chromatic partitions with $k$ colours, respectively.

Definition 3. An $(l, k)$-chromatic overpartition of $n$ is an overpartition of $n$, where each nonoverlined part can take up to $k$ colours and every first occurrence of any part may appear overlined with $l \leq k$ distinct colours. Equal parts with different colours shall be considered distinct. 


\section{Example 2.}

- $\overline{5}_{3}+\overline{5}_{1}+4_{3}+\overline{2}_{1}+2_{1}+2_{0}+1$ is a (5,6)-chromatic overpartition of 21 different from $\overline{5}_{1}+\overline{5}_{3}+4_{3}+\overline{2}_{1}+2_{1}+2_{0}+1$.

- For $n=k=l=4,4_{a}, \overline{4}_{a}, 3_{a}+1_{b}, \overline{3}_{a}+1_{b}, 3_{a}+\overline{1}_{b}, \overline{3}_{a}+\overline{1}_{b}, 2_{a}+2_{b}, \overline{2}_{a}+2_{b}, 2_{a}+1_{b}+1_{c}$, $\overline{2}_{a}+1_{b}+1_{c}, 2_{a}+\overline{1}_{b}+1_{c}, \overline{2}_{a}+\overline{1}_{b}+1_{c}, 1_{a}+1_{b}+1_{c}+1_{d}, \overline{1}_{a}+1_{b}+1_{c}+1_{d}$ are indexed overpartitions, taking $0 \leq a \leq 3,0 \leq b \leq 3,0 \leq c \leq 3$ and $0 \leq d \leq 3$, we have the 872 chromatic overpartitions of $n=4$ with four colours.

Denote the number of $(l, k)$-chromatic overpartitions of $n$ by $\bar{p}_{l, k}(n)$. The next table show a comparative between $\bar{p}(n)$ and $\bar{p}_{n, n}(n)$ for some values of $n$.

\begin{tabular}{|c|c|c|}
\hline \hline$n$ & $\bar{p}(n)$ & $\bar{p}_{n, n}(n)$ \\
\hline \hline 1 & 2 & 2 \\
\hline 2 & 4 & 12 \\
\hline 3 & 8 & 96 \\
\hline 4 & 14 & 872 \\
\hline 5 & 24 & 9960 \\
\hline 6 & 40 & 138180 \\
\hline 7 & 60 & 2296644 \\
\hline \hline
\end{tabular}

Table 2. Comparison between the number of overpartitions and the number of chromatic overpartitions

Definition 4. An $(n, k)$-super-chromatic overpartition is an overpartition of $n$ where each part $j$ can appear with $k^{j}$ different colours, and the order of equal coloured parts matters.

Example 3. $\overline{5_{82}}+4_{7}+\overline{2_{0}}+2_{3}+2_{0}+1_{0}$ is a $(16,3)$-super-chromatic overpartition. The chromatic overpartitions $4_{0}+4_{1}+\overline{2_{0}}+1_{2}$ is different from $4_{1}+4_{0}+\overline{2_{0}}+1_{2}$, as an $(11,3)$-chromatic overpartition.

Definition 5. An $(n, k, 2)$-chromatic partition is a partition of $n$ with two indexes where the first ascribes colours from 0 to $k-1$, the second index is a number 0 or 1 . For equal parts and equal first indexes, the order of sum of this parts should be disregarded. In other words, for a sum of parts $j_{a, i}+j_{a, l}$ is the same as $j_{a, l}+j_{a, i}$ but $j_{a, i}+j_{b, l}$ is different from $j_{b, i}+j_{a, l}$ for $a \neq b$ in $\{0,1, \ldots, k-1\}$, and $i, j \in\{0,1\}$.

Example 4. $5_{0,1}+4_{2,1}+3_{1,0}+3_{1,1}+2_{1,1}+1_{0,0}+1_{2,0}+1_{2,0}$ is a $(20,3,2)$-chromatic partition.

Definition 6. An $(n, k, 2)$-super-chromatic partition is a $(n, k)$-super-chromatic partition that appears with two indices, where the first ascribes colours from 0 to $k-1$, the second index is a number 0 or 1 . For equal parts and equal first index, the order of sum of this parts should be disregarded. 
Definition 7. An ( $n, k, 2)$-super-chromatic partition of type $A$ is an $(n, k, 2)$-super-chromatic partition, where:

- the even parts have the second index equal to 0 ;

- the odd parts have index equal to 0 or 1.

Example 5. The partition $5_{40,1}+5_{40,0}+4_{15,1}+3_{7,0}+2_{5,1}+1_{0,0}+1_{0,1}$ is a $(21,2,2)$-superchromatic partition, and $5_{40,1}+3_{6,1}+3_{7,0}+2_{5,1}+1_{0,0}+1_{0,1}$ is a $(15,2,2)$-super-chromatic partition of type $A$.

Definition 8. Let $k$ be a perfect square. An $(n, k, 2)$-chromatic partition of type $A$ is an $(n, k, 2)$ chromatic partition, where:

- the even parts appear up to $\sqrt{k}$ distinct colours, with second index equal to 1 ;

- the odd parts appear up to $k$ colours with first index equal to 0 , and with the second index equal to 1 , the odd parts can take up to $\sqrt{k}$ colours.

Proposition 1. The generating function for the number of $(1, k)$-chromatic partitions with distinct parts is

$$
(-k q, q)_{\infty}
$$

Furtermore, the product

$$
(-k q, k q)_{\infty}
$$

is the generating function for the number of $(1, k)$-super-chromatic partitions with distinct parts.

Proof. Expanding $(-k q, q)_{\infty}$ and $(-k q, k q)_{\infty}$, we have

$$
(1+k q)\left(1+k q^{2}\right)\left(1+k q^{3}\right) \ldots\left(1+k q^{j}\right) \ldots
$$

and

$$
(1+k q)\left(1+k^{2} q^{2}\right)\left(1+k^{3} q^{3}\right) \ldots\left(1+k^{j} q^{j}\right) \ldots
$$

respectively.

Every choice in the first product can be associated with a partition with distinct parts, each part having $k$ colours at most. In the second, every distinct part $j$ may appear with $k^{j}$ distinct colours.

Combining the products above, and taking into consideration that the coloured overlined parts are distinct, we have the next result.

Corollary 1. The infinite products:

$$
\frac{(-l q, q)_{\infty}}{(k q, q)_{\infty}} \text { and } \quad \frac{(-k q, k q)_{\infty}}{(k q, k q)_{\infty}}
$$

are the generating functions for the number of the $(l, k)$-chromatic overpartitions and superchromatic overpartitions with $k$ colours, respectively. 


\section{Proofs using generating functions}

In the next two theorems we will provide interpretations using chromatic overpartitions for some identities. The proofs are made using only cancellations laws.

Theorem 1. Let $k$ be a perfect square. The number of $(\sqrt{k}, k)$-chromatic overpartitions of $n$ is equal to the number of $(n, k, 2)$-chromatic partitions of type $A$.

Proof. Taking the generating function for $(\sqrt{k}, k)$-chromatic overpartitions of $n$, we have

$$
\begin{gathered}
\frac{(-\sqrt{k} q, q)_{\infty}}{(k q, q)_{\infty}}=\frac{(1+\sqrt{k} q)\left(1+\sqrt{k} q^{2}\right)\left(1+\sqrt{k} q^{3}\right) \cdots\left(1+\sqrt{k} q^{n}\right) \cdots}{(1-k q)\left(1-k q^{2}\right)\left(1-k q^{3}\right) \cdots\left(1-k q^{n}\right) \cdots} \\
=\frac{1}{(1-k q)(1-\sqrt{k} q)\left(1-\sqrt{k} q^{2}\right)\left(1-k q^{3}\right)\left(1-\sqrt{k} q^{4}\right) \cdots\left(1-k q^{n}\right)\left(1-k q^{5}\right)\left(1-\sqrt{k} q^{5}\right) \cdots} \\
=\prod_{\substack{n o d d \\
j \in \mathbb{Z}_{+}}} \frac{1}{\left(1-k q^{n}\right)\left(1-\sqrt{k} q^{j}\right)}=\frac{1}{(\sqrt{k} q, q)_{\infty}\left(k q, q^{2}\right)_{\infty}},
\end{gathered}
$$

which is the generating function for $(n, k, 2)$-chromatic partitions of type $A$.

Theorem 2. The number of $(n, k)$-super-chromatic overpartitions is equal to the number of $(n, k, 2)$-super-chromatic partitions of type $A$.

Proof. Considering the generating function for $k$-super chromatic overpartitions

$$
\begin{gathered}
\frac{(-k q, k q)_{\infty}}{(k q, k q)_{\infty}}=\frac{(1+k q)\left(1+k^{2} q^{2}\right)\left(1+k^{3} q^{3}\right) \cdots\left(1+k^{n} q^{n}\right) \cdots}{(1-k q)\left(1-k^{2} q^{2}\right)\left(1-k^{3} q^{3}\right) \cdots\left(1-k^{n} q^{n}\right) \cdots} \\
=\frac{1}{(1-k q)^{2}\left(1-k^{2} q^{2}\right)\left(1-k^{3} q^{3}\right)^{2}\left(1-k^{4} q^{4}\right)\left(1-k^{5} q^{5}\right)^{2} \cdots} \\
=\frac{1}{(k q, k q)_{\infty}\left(k q, k q^{2}\right)_{\infty}},
\end{gathered}
$$

where the last expression is the generating function for $(n, k, 2)$-super-chromatic partitions of type $A$.

\section{The bijective proofs}

\section{Bijective proof for Theorem 1.}

Let $C_{n}(\sqrt{k}, k)$ be the set of all $(\sqrt{k}, k)$-chromatic overpartitions of $n$, and $A_{n}(k, 2)$ be the set of $(n, k, 2)$-chromatic partitions of type $A$. Let $f: C_{n}(\sqrt{k}, k) \rightarrow A_{n}(k, 2)$ be a function given by:

- non-overlined odd parts are associated with the same odd part with equal first index and second index equal to 0 ;

- overlined odd parts are associated with the same odd part with equal first index and second index equal to 1 ; 
- overlined even parts are associated with pairs of parts with equal first index and second index equal to 1 ;

- a non-overlined even part $\alpha_{l}, 0 \leq l \leq \sqrt{k}-1$ is associated with the same part with $\alpha_{l, 1}$;

- a non-overlined even part $\alpha_{l}, \sqrt{k} \leq l \leq k-1$, where $\alpha=2 \lambda$ is associated with the sum of parts $\lambda_{i, 1}+\lambda_{j, 1}$, for $i, j \in\{0,1, \ldots, \sqrt{k}-1\}$.

We claim that the function $f$ is bijective. In fact, the first two conditions establish associations 1-1 between odd parts of overpartitions, either overlined or not, and their corresponding in biindexed partitions. The last three conditions refer to the overpartitions in even parts. The latter associates the part $\alpha_{l}$ with the sum of parts $(\alpha / 2)_{i, 1}+(\alpha / 2)_{j, 1}$ for $i, j \in\{0,1, \ldots, \sqrt{k}-1\}$. Indeed, this latter association is $1-1$, since, by the multiplicative principle, there is $\sqrt{k} \cdot \sqrt{k}=k$ bi-indexed partitions of type $(\alpha / 2)_{i, 1}+(\alpha / 2)_{j, 1}$, and therefore, the function $f$ is bijective.

Example 6. For instance, $f\left(\overline{2_{1}}+\overline{1_{0}}\right)=1_{1,1}+1_{1,1}+1_{0,1}, f\left(2_{2}+\overline{1_{1}}\right)=1_{0,1}+1_{1,1}+1_{1,1}$, and $f\left(\overline{4_{0}}+\overline{3_{0}}+2_{2}+2_{2}\right)=2_{0,1}+2_{0,1}+3_{1,1}+1_{0,1}+1_{1,1}$.

The next table shows the effect of the function $f: C_{3}(2,4) \rightarrow A_{3}(4,2)$ in parts of chromatic overpartitions and $(3,4,2)$-chromatic partitions.

\begin{tabular}{|c|c|c|c|c|c|}
\hline \hline $1_{0}$ & $\leftrightarrow$ & $1_{0,0}$ & $2_{3}$ & $\leftrightarrow$ & $1_{1,1}+1_{0,1}$ \\
\hline $1_{1}$ & $\leftrightarrow$ & $1_{1,0}$ & $\overline{2_{0}}$ & $\leftrightarrow$ & $1_{0,1}+1_{0,1}$ \\
\hline $1_{2}$ & $\leftrightarrow$ & $1_{2,0}$ & $\overline{2_{1}}$ & $\leftrightarrow$ & $1_{1,1}+1_{1,1}$ \\
\hline $1_{3}$ & $\leftrightarrow$ & $1_{3,0}$ & $3_{0}$ & $\leftrightarrow$ & $3_{0,0}$ \\
\hline$\overline{1_{0}}$ & $\leftrightarrow$ & $1_{0,1}$ & $3_{1}$ & $\leftrightarrow$ & $3_{1,0}$ \\
\hline$\overline{1_{1}}$ & $\leftrightarrow$ & $1_{1,1}$ & $3_{2}$ & $\leftrightarrow$ & $3_{2,0}$ \\
\hline $2_{0}$ & $\leftrightarrow$ & $2_{0,1}$ & $3_{3}$ & $\leftrightarrow$ & $3_{3,0}$ \\
\hline $2_{1}$ & $\leftrightarrow$ & $2_{1,1}$ & $\overline{3_{0}}$ & $\leftrightarrow$ & $3_{0,1}$ \\
\hline $2_{2}$ & $\leftrightarrow$ & $1_{0,1}+1_{1,1}$ & $\overline{3_{1}}$ & $\leftrightarrow$ & $3_{1,1}$ \\
\hline \hline
\end{tabular}

Table 3. Example of bijective association between parts of overpartitions and parts of chromatic partitions of type $A$.

\section{Bijective proof for Theorem 2.}

For $n$ and $k$ positive integers, denote the set of all super-chromatic overpartitions of $n$ in $k$ colours by $S_{n}(k)$, and denote by $S_{n}(k, 2)$ the set of $(n, k, 2)$-super-chromatic partitions of type $A$. Let $g: S_{n}(k) \rightarrow S_{n}(k, 2)$ be a function given by:

- non-overlined odd parts are associated with the same part with equal first index and second index equal to 0 ;

- overlined odd parts are associated with the same part with equal first index and second index equal to 1 ; 
- non-overlined even parts are associated with the same part with equal first index and second index equal to 0 ;

- an overlined even part $\overline{\lambda_{l}}$ is associated with a sum of the kind $1_{i_{1}, 1}+1_{i_{2}, 1}+\cdots+1_{i_{l}, 1}$, where $i_{1}, i_{2}, \cdots, i_{l} \in\{0,1\}$, in the following order $\overline{\lambda_{0}} \leftrightarrow 1_{0,1}+1_{0,1}+\cdots+1_{0,1}, \overline{\lambda_{1}} \leftrightarrow$ $1_{1,1}+1_{0,1}+\cdots+1_{0,1}$, and so on.

The first condition makes a bijective association between odd parts of super-chromatic overpartitions of type $\lambda_{l}$ and parts $\lambda_{l, 0}$ of $(n, k, 2)$-super-chromatic partitions of type $A$, as well as the second and third conditions make the bijective associations $\overline{\alpha_{l}} \leftrightarrow \alpha_{l, 1}$, and $\beta_{l} \leftrightarrow \beta_{l, 0}$, respectively, for an odd number $\alpha$ and an even number $\beta$.

For the last class of chromatic overpartitions, an overlined part $\overline{\lambda_{l}}$ is associated with a biindexed partition of type $1_{i_{1}, 1}+1_{i_{2}, 1}+\ldots+1_{i_{l}, 1}$. Since each $i_{j}$ index refers to one of the $k$ colours available, by the multiplicative principle, there are $k^{\lambda}$ partitions of this type, and since we are associating each one of them with super-chromatic overpartitions, we obtain the desired 1-1 association.

For parts in $\{1,2,3\}$ the next table shows the the 1-1 association between super-chromatic overpartitions and parts of super-chromatic partitions of type $A$.

\begin{tabular}{|c|c|c|c|c|c|}
\hline \hline $1_{0}$ & $\leftrightarrow$ & $1_{0,0}$ & $3_{2}$ & $\leftrightarrow$ & $3_{2,0}$ \\
\hline $1_{1}$ & $\leftrightarrow$ & $1_{1,0}$ & $3_{3}$ & $\leftrightarrow$ & $3_{3,0}$ \\
\hline$\overline{1_{0}}$ & $\leftrightarrow$ & $1_{0,1}$ & $\overline{2_{1}}$ & $\leftrightarrow$ & $3_{4,0}$ \\
\hline$\overline{1_{1}}$ & $\leftrightarrow$ & $1_{1,1}$ & $3_{0}$ & $\leftrightarrow$ & $3_{5,0}$ \\
\hline $2_{0}$ & $\leftrightarrow$ & $2_{0,0}$ & $3_{6}$ & $\leftrightarrow$ & $3_{6,0}$ \\
\hline $2_{1}$ & $\leftrightarrow$ & $2_{1,0}$ & $3_{7}$ & $\leftrightarrow$ & $3_{7,0}$ \\
\hline $2_{2}$ & $\leftrightarrow$ & $2_{2,0}$ & $\overline{3_{0}}$ & $\leftrightarrow$ & $3_{0,1}$ \\
\hline $2_{3}$ & $\leftrightarrow$ & $2_{3,0}$ & $\overline{3_{1}}$ & $\leftrightarrow$ & $3_{1,1}$ \\
\hline$\overline{2_{0}}$ & $\leftrightarrow$ & $1_{0,1}+1_{0,1}$ & $\overline{3_{1}}$ & $\leftrightarrow$ & $3_{2,1}$ \\
\hline$\overline{2_{1}}$ & $\leftrightarrow$ & $1_{0,1}+1_{1,1}$ & $\overline{3_{1}}$ & $\leftrightarrow$ & $3_{3,1}$ \\
\hline$\overline{2_{2}}$ & $\leftrightarrow$ & $1_{1,1}+1_{0,1}$ & $\overline{3_{1}}$ & $\leftrightarrow$ & $3_{4,1}$ \\
\hline$\overline{2_{3}}$ & $\leftrightarrow$ & $1_{1,1}+1_{1,1}$ & $\overline{3_{1}}$ & $\leftrightarrow$ & $3_{5,1}$ \\
\hline $3_{0}$ & $\leftrightarrow$ & $3_{0,0}$ & $\overline{3_{6}}$ & $\leftrightarrow$ & $3_{6,1}$ \\
\hline $3_{1}$ & $\leftrightarrow$ & $3_{1,0}$ & $\overline{3_{7}}$ & $\leftrightarrow$ & $3_{7,1}$ \\
\hline \hline
\end{tabular}

Table 4. Bijective association between parts of super-chromatic overpartitions and parts of super-chromatic partitions of type $A$.

Example 7. There are sixty-eight super-chromatic overpartitions of $n=3$ with $k=2$ colours. Indeed for $s_{1}=3_{0}, s_{2}=2_{1}+1_{0}, s_{3}=\overline{2_{2}}+1_{0}$, and $s_{4}=\overline{1_{0}}+1_{0}+1_{0}$, the correspondent $(3,2,2)$-chromatic partition are $g\left(s_{1}\right)=3_{0,0}, g\left(s_{2}\right)=2_{1,0}+1_{0,0}, g\left(s_{3}\right)=1_{2,1}+1_{2,1}+10,0$ and $g\left(s_{4}\right)=1_{0,1}+1_{0,0}+1_{0,0}$, respectively.

$A(12,3,2)$-super-chromatic partitions of type $A, 4_{5,0}+2_{3,1}+20,1+1_{1,1}+1_{0,1}+1_{0,1}+1_{0,1}$ is associated to $g^{-1}\left(4_{5,0}+2_{3,1}+20,1+1_{1,1}+1_{0,1}+1_{0,1}+1_{0,1}\right)=4_{5}+\overline{4_{1}}+2_{3}+2_{0}$. 


\section{References}

[1] Alegri, M. Combinatorial Interpretations for Identities involving Chromatic Partitions, submitted.

[2] Andrews, G. E., \& Eriksson, K. (2004). Integer Partitions, Cambridge University Press.

[3] Bressoud, D. M. (1978). A new family of partition identities, Pacific J. Math., 77, 71-74.

[4] Bressoud, D. M. (1981). Some identities for terminating $q$-series, Math. Proc. Cambridge Phil. Soc., 211-223.

[5] Lovejoy, J. (2004). Overpartitions, Trans. Amer. Math. Soc., 356, 1623-1635.

[6] Lovejoy, J. (2006). Overpartitions Pairs, Ann. Inst. Fourier (Grenoble), 56, 781-794.

[7] Lovejoy, J. (2007). Partitions and overpartitions with attached parts, Arch. Math., 88, 316322.

[8] Pak, I. (2006). Partition Bijections, a Survey, Ramanujan Journal, 12, 5-75. 